\title{
Extreme Arteriendurchmesser erhöhen vaskuläres Risiko
}

Engstellen in Arterien sind ein Hinweis für drohende vaskuläre Komplikationen. Auch Ektasien und Aneurysmen haben einen Krankheitswert, der aber weit weniger gut erforscht ist. Dabei scheinen sich sowohl auffällig enge als auch weite Arterien als Biomarker für kardiovaskuläre Ereignisse zu eignen.

In einer aktuellen Studie wurden die Daten von 1034 Teilnehmern der 1993 begonnenen NOMAS-Studie analysiert, die eine Time-of-flight-Magnetresonanz-
Angiografie erhalten hatten. Für jeden Probanden wurden anhand der 3-dimensionalen Rekonstruktion zunächst die Durchmesser von bis zu 13 Hirnarterien in Form eines Z-Scores errechnet. Den Mittelwert über alle Hirnarterien bezeichneten die Autoren als Brain-arterialremodelling-Score (BAR-Score). Ein BAR- 
Score von $<-2$ galt als besonders kleiner, ein Score von $>2$ als besonders großer Gefäßdurchmesser. Endpunkte waren

- Gesamtmortalität,

- Schlaganfall und

- kardiovaskuläre Ereignisse.

Das Durchschnittsalter der Patienten lag bei 70 Jahren. 75\% litten an Bluthochdruck, 25\% an Diabetes, 68\% an Hypercholesterinämie und $16 \%$ rauchten. Die mittlere Nachbeobachtungszeit betrug 8 Jahre. Im Vergleich zu den Probanden mit normalen Hirnarteriendurchmessern hatten Patienten mit besonders engen bzw. weiten Arterien ein höheres Risiko für
- $\operatorname{Tod}(\mathrm{p}=0,001)$,

- vaskulären Tod $(\mathrm{p}=0,02)$,

- ein vaskuläres Ereignis jeglicher Art $(\mathrm{p}=0,05)$ und

- einen Herzinfarkt $(\mathrm{p}=0,10)$.

Bei den Teilnehmern mit den engsten Arterien war zusätzlich noch das Risiko für einen ischämischen Schlaganfall erhöht. Sie hatten zudem eine doppelt so hohe Gesamt- und kardiovaskuläre Mortalität wie Probanden mit normokalibrigen Hirnarterien. Patienten mit besonders weiten Arterien hatten hingegen eine $3 \times$ höhere kardiovaskuläre Mortalität und Herzinfarktrate.
Offenbar erhöhen sowohl überdurchschnittlich enge als auch weite Hirnarterien das individuelle Risiko für vaskuläre Ereignisse, folgern die Autoren. Sie werten die Ergebnisse als Hinweis auf eine nichtlineare Beziehung zwischen dem systemischen und zerebralen Gefäßsystem.

Dr. med. Susanne Meinrenken, Bremen DOI 10.1055/s-0041-105507

Gutierrez et al. Brain arterial diameters ... J Am Heart Assoc 2015; 4: e002289 